\title{
Médiévales
}

Langues, Textes, Histoire

47 | automne 2004

Îles du Moyen Âge

\section{Les îles dans les descriptions géographiques et les cartes du Moyen Âge}

Islands in the Geographical Descriptions and Maps in the Middle Ages

\section{Nathalie Bouloux}

\section{(2) OpenEdition}

12 Journals

Édition électronique

URL : https://journals.openedition.org/medievales/502

DOI : 10.4000/medievales.502

ISSN : 1777-5892

Éditeur

Presses universitaires de Vincennes

Édition imprimée

Date de publication : 1 décembre 2004

Pagination : 47-62

ISBN : 2-84292-157-7

ISSN : 0751-2708

\section{Référence électronique}

Nathalie Bouloux, "Les îles dans les descriptions géographiques et les cartes du Moyen Âge »,

Médiévales [En ligne], 47 | automne 2004, mis en ligne le 02 septembre 2006, consulté le 22 avril 2022.

URL : http://journals.openedition.org/medievales/502; DOI : https://doi.org/10.4000/medievales.502

Ce document a été généré automatiquement le 22 avril 2022.

Tous droits réservés 


\title{
Les îles dans les descriptions géographiques et les cartes du Moyen Âge
}

Islands in the Geographical Descriptions and Maps in the Middle Ages

\author{
Nathalie Bouloux
}

1 Dans les textes géographiques comme sur les cartes du Moyen Âge, les îles peuvent être un lieu du prodige. Ce lien entre îles et merveilles a retenu depuis longtemps l'attention des historiens et des spécialistes de la littérature médiévale. Les îles sont dès lors interprétées comme l'archétype d'une perception symbolique de l'espace, qui serait propre au Moyen Âge : entre réel et imaginaire, entre terre et mer, image même de l'entre-deux, elles ne laissent place qu'à la fantaisie et au fabuleux, surtout lorsqu'elles sont situées dans l'espace mouvant de l'anneau océanique. Les îles méditerranéennes, forcément mieux connues, sont elles chargées d'histoire et de souvenirs antiques ${ }^{1}$. Mais percevoir l'intérêt que les lettrés médiévaux portent aux phénomènes insulaires comme relevant seulement de leur propension à accueillir l'imaginaire ou, pire, de leur incapacité à concevoir rationnellement l'espace est réducteur. Les îles ont une place dans les descriptions du monde. Elles sont aussi l'objet d'interrogations, d'investigations et de représentations. En analysant leur rôle dans les descriptions de l'espace, il est possible d'approcher la manière dont le monde insulaire était perçu, pensé et représenté dans la culture géographique du Moyen Âge. Pour cela, il conviendra de réfléchir sur la place des îles dans l'économie des descriptions géographiques, d'étudier la perception de leur nature et de leurs fonctions et de s'interroger sur les modalités de leur représentation cartographique.

La place des îles dans les descriptions géographiques

2 On a souvent constaté que les îles recevaient un traitement particulier dans les textes géographiques médiévaux, soit qu'elles ferment le texte, soit qu'elles constituent une section spécifique, toujours sous la forme d'un catalogue ou d'une liste plus ou moins ordonnés spatialement. À partir de quand ce procédé descriptif apparaît-il dans les textes? Dans l'Antiquité, certains géographes avaient l'habitude de les traiter à part du 
continent, comme Strabon et Ptolémée ${ }^{2}$. Mais ces auteurs, inconnus du Moyen Âge latin, n'ont pu influencer les auteurs médiévaux.

On s'en tiendra ici aux auteurs antiques connus durant la période médiévale, ceux dont la lecture informe les lettrés médiévaux. Dans les livres III à VI de l'Histoire naturelle, Pline donne une description exhaustive du monde romain. Il y intègre les îles dans le cours de l'exposé en leur consacrant un paragraphe lors de la description des mers, ou en les insérant lors de l'évocation du littoral. Chez Pline, les îles se fondent dans la description, sans qu'une place particulière leur soit réservée ${ }^{3}$. L'Histoire naturelle propose également un modèle de description, repris par les auteurs médiévaux : traiter d'une île impose d'en donner l'orientation, d'indiquer la distance qui la sépare de la côte ou d'autres îles ainsi que la mesure de sa circonférence, de fournir quelques détails sur son contenu (villes, phénomènes naturels, particularités) ${ }^{4}$. On observe la même disposition dans les Collectanea rerum memorabilium de Solin, avec une tendance propre à l'auteur, d'insister sur les particularités insulaires (mentions d'îles méditerranéennes dépourvues de serpents, remarque sur la multitude des îles dans les parages de l'Irlande et qualification de la Bretagne comme un alter orbis). Pomponius Mela traite des îles dans un chapitre à part (îles de la mer intérieure, îles océaniques ${ }^{5}$ ) mais il est peu connu jusqu'au XIV ${ }^{\mathrm{e}}$ siècle. Dans les descriptions des géographes latins qui ont servi de fondement au savoir géographique des lettrés médiévaux, les îles ne sont donc pas placées dans une section à part mais sont décrites selon des modalités adaptées à leur nature.

Orose, dans le chapitre de ses Histoires contre les païens dédié à la description du monde leur consacre une place spécifique. Les îles du Nord-Ouest sont décrites après la Gaule et l'Espagne, les Fortunées sont insérées à la fin de la description de l'Afrique, Taprobane à la suite de l'Inde. Ce sont surtout les îles méditerranéennes qui forment une section clairement individualisée : «Je vais à présent délimiter l'emplacement, le nom et l'étendue des îles qui sont dans notre mer $»^{6}$. Le tableau géographique d'Orose sert de modèle à la plupart des descriptions géographiques médiévales parce qu'il fournit un ordre descriptif, celui d'un découpage ordonné du monde connu selon trois ensembles, l'Asie, l'Europe, et l'Afrique. À l'intérieur de chacune de ces parties, les régions sont définies et localisées par leur contiguïté, mettant ainsi en évidence l'ordonnancement du monde pour en faciliter la mémorisation. Les îles, qui n'ont d'autres contiguïtés que la mer, trouvent difficilement place dans ce système: leur réserver un chapitre particulier pallie ce défaut.

Isidore de Séville est le second grand modèle de la géographie médiévale. C'est à lui que revient l'étymologie de l'île "Insulae dictae quod in salo sint, id est in mari ${ }^{7}$ ", si souvent reprise, y compris des savants de la Renaissance qui semblent en avoir oublié l'origine. L'île est donc un monde clos par la mer, mais un espace terrestre. En effet, les îles sont incluses dans le livre XIV des Étymologies consacré à l'élément terre tandis que l'eau est le sujet du livre XIII. Il n'est pas sans intérêt d'étudier leur place dans le livre XIV : après avoir donné les étymologies de "mundo » et de " terra ", l'évêque de Séville décrit le monde connu selon le schéma d'Orose, sa source principale. Il ajoute ensuite quatre sections : îles, promontoires, montagnes et les inferiora. Chacune de ces sections est un catalogue, qui pourrait faire penser que l'on atteint ici le degré zéro de la géographie, si ce n'est que son établissement implique au moins un acte de classification, c'est-à-dire de définition et d'identification. On ajoutera que le catalogue des îles est ordonné spatialement (îles océaniques, îles du Nord-Ouest, îles africaines, 
îles du Sud-Est, puis îles méditerranéennes selon un ordre allant de l'est à l'ouest). Encore l'identification de la nature insulaire n'est-elle pas aussi simple: il existe en effet une relation analogique entre l'île et le promontoire, et par conséquent entre l'île et la montagne comme l'indiquent les phrases liminaires des sections sur les promontoires et sur les montagnes ${ }^{8}$. Est commun aux îles, promontoires et montagnes leur jaillissement hors d'un espace perçu comme plan. L'analogie existe aussi entre l'île entourée d'eau et le promontoire s'avançant dans l'eau, qui constituent deux formes de contact entre terre et mer. Cette ressemblance de nature justifie l'intégration des presqu'îles, comme par exemple l'Achaïe, dans le catalogue des îles - comme l'avait fait Orose avant Isidore et comme tant d'autres le feront après lui. Comme objets géographiques singuliers, montagnes, îles et promontoires doivent être rassemblés dans des sections dont la succession, qui s'achève sur les inferiora (dépressions, espaces souterrains, gorges, etc.), fait sens. Ce procédé analytique découle aussi d'un principe de l'ordre du savoir : pour bien savoir les choses, il faut en savoir le détail. Ce qui est habituellement présenté comme un procédé de description propre à l'espace insulaire ressort d'une méthode de la géographie médiévale, et s'applique également aux fleuves, aux mers, aux peuples ${ }^{9} .$. On ajoutera enfin qu'Isidore de Séville ne prétendait pas à l'exhaustivité mais a choisi de signaler les îles qui, par leur importance ou par leur singularité, méritaient plus particulièrement d'être mémorisées.

6 Le choix de classer les îles à part répond donc à un problème posé à ceux qui entendent décrire l'espace : insérer dans un cadre descriptif fondé sur la délimitation des régions un objet essentiellement mouvant; rendre compte de leur intérêt comme élément spécifique de la représentation de l'espace. À quelques exceptions près, ce procédé est retenu par les auteurs médiévaux, non sans aménagements et évolutions dus en particulier à l'intérêt croissant pour la mer. Deux exemples nous retiendront ici.

$7 \mathrm{Au} \mathrm{XII}{ }^{\mathrm{e}}$ siècle, Hugues de Saint-Victor réserve une place importante aux îles dans sa Descriptio mappe mundi, dont il convient de rappeler qu'elle est le résultat écrit du commentaire oral d'une mappemonde, et procède d'un renouvellement majeur dans les représentations de l'espace ${ }^{10}$. Le commentaire, à caractère pédagogique, commence par la description de l'anneau océanique et de son contenu insulaire, puis se prolonge par celle de la Méditerranée et de ses principales îles. Une section originale consacrée aux cités de quelques-unes des îles les plus importantes est insérée. Cette première partie du commentaire s'achève sur la mer Rouge. Ensuite, les régions sont minutieusement décrites, sur le modèle d'Orose. À la fin du traité, sont ajoutées une section sur la Bretagne, une sur l'Irlande et une dernière intitulée " De situ quarumdam insularum », toutes trois fondées sur Orose. Ces ajouts pourraient faire penser à un intérêt particulier pour les îles s'ils n'étaient accompagnés de descriptions de la péninsule ibérique, elle aussi issue d'Orose, et de la Gaule et de l'Italie, prises dans l'Historia ecclesiastica d'Hugues de Fleury. Ces passages tranchent par leur origine exclusivement livresque, alors que le reste de la Descriptio est le commentaire d'une carte: il est probable que leur présence tient plus au désir de l'auteur d'amplifier sa description des régions du cœur de l'Occident latin qu'à un intérêt particulier pour les îles ${ }^{11}$.

8 En revanche, le traitement réservé aux îles dans la partie issue du commentaire du maître est remarquable. Hugues de Saint-Victor se distingue par le souci de donner une localisation précise des îles: pour les îles océaniques, il utilise les vents ${ }^{12}$ tandis que celles de la Méditerranée sont situées par rapport aux terres suivant un itinéraire rigoureux ${ }^{13}$. Surtout, leur insertion dans le cours de la description est rendue possible 
par la place réservée aux mers, qui tient tout à la fois de la nature du traité commentaire d'une carte - et de la promotion des arts mécaniques - en particulier des arts de la navigation. Il faut néanmoins nuancer la part du maritime dans la description de l'île par l'insistance sur sa nature terrestre : l'île, par définition située dans l'eau, est un espace de terre conquis par l'homme comme en témoigne la conclusion du paragraphe consacré aux îles de la mer Adriatique : «Parmi ces îles, qui sont au nombre de 49 , on trouve cinq îles principales, plusieurs ont des cités voire des régions ${ }^{14} »$. Enfin, une bizarrerie mérite d'être signalée: dans les îles de l'Adriatique, il inclut curieusement "Venetia, Brundusium, Apulia »" ${ }^{15}$ L'île relève d'une double nature, à la fois maritime et terrestre.

9 Barthélemy l'Anglais, dans son De proprietatibus rerum, choisit d'inclure les îles dans la section consacrée aux régions. Mais il est encyclopédiste, certes remarquablement informé et « moderne » dans ses descriptions, mais plus soucieux de donner accès à des informations concrètes, classées alphabétiquement (pour la première fois) que de procurer une description ordonnée du monde. Il a pu lui sembler expédient de regrouper les îles avec les régions, d'autant qu'il retient essentiellement celles qui sont suffisamment grandes pour leur être assimilées. Pour justifier son choix, il donne une définition générique d'«insula» dans laquelle, après avoir rappelé l'étymologie isidorienne, il insiste sur le caractère terrestre de l'île et oppose l'intérieur, résolument terrien, aux rivages mordus par les eaux ${ }^{16}$.

10 Pourtant, c'est bien la composante maritime des îles qui explique la part croissante qu'elles prennent dans les descriptions, au point de devenir le sujet principal du De insulis (vers 1385-1389) du notaire florentin Domenico Silvestri et du Liber insularum archipelagi (vers 1420-1422) de Cristoforo Buondelmonti. Quelques témoins préfigurent cet intérêt. En premier lieu, songeons aux nombreux textes qui, dès le $\mathrm{xII}^{\mathrm{e}}$ siècle, mentionnent les itinéraires maritimes des expéditions de croisade, et aux ancêtres des portulans. Les descriptions des littoraux font évidemment une large place aux îles, comme escales et comme relais, avec parfois une évocation rapide de leur situation et de leurs habitants. Rapidement, ce savoir technique, expression de la culture des hommes de la mer, entre dans les sources de la connaissance de l'espace des lettrés ${ }^{17}$.

$11 \mathrm{Au}$ début du XIV siècle, le notaire ferrarais Riccobaldus compose une description du monde qu'il intitule: De locis orbis et insularum et marium. Historien venu progressivement à la nécessité de décrire le monde pour en comprendre l'histoire, novateur dans son souci de reconstituer l'espace de l'Antiquité, Riccobaldus se distingue aussi dans la part qu'il réserve au monde insulaire, comme l'atteste le plan suivi : présentation générale du monde, description des trois parties sur le modèle d'Orose, puis des îles méditérranéennes et océaniques, troisième partie au contenu hétéroclite (régions de l'Italie, énumération des fleuves et des montagnes de l'orbis). Si l'on exclut la troisième partie, au caractère inachevé et que rien n'annonce dans le titre, il apparaît que sa description du monde est en réalité bipartite : d'un côté l'orbis, de l'autre l'espace maritime et ses îles. Cet intérêt pour les mers est sans doute une conséquence de l'explosion commerciale - certes déjà ancienne - en Méditerranée, renforcée par la diffusion de nouveaux outils d'appréhension intellectuelle de l'espace maritime, le portulan et la carte marine ${ }^{18}$. Dans le De locis, on remarquera la mention de deux îles que les marins appellent «Duas Sorores", qui vient de la lecture d'une carte marine et s'ajoute ainsi aux îles connues par la tradition livresque. Un des effets de la diffusion des cartes marines est l'intégration dans la représentation du monde d'îles 
non citées par les Anciens. Un autre texte vient appuyer cette remarque. Il s'agit d'une description intitulée "De insulis minoribus", jointe au dossier cartographique qui accompagne le Liber secretorum fidelium crucis de Marino Sanudo (début du XIV e siècle). Ce texte, qui commence par une réflexion sur la représentation cartographique, est conçu comme un complément de la mappemonde qui, en raison de sa taille, donne à voir essentiellement les îles grandes et fameuses, connues à travers les descriptions traditionnelles du monde ${ }^{19}$. L'ajout du «De insulis minoribus» montre la nécessité d'évoquer un monde insulaire partiellement ignoré des descriptions de l'espace, et principalement connu par les portulans et les cartes marines. Ajoutons enfin que la contemplation des cartes - mappemondes et cartes marines - comme la lecture des récits de voyageurs qui se diffusent alors, renvoient aux remarques des géographes antiques concernant le nombre étonnant des îles en certaines régions du monde. Lorsque Domenico Silvestri présente le projet de son De insulis, il s'émerveille et se désole qu'un si grand nombre d'îles, pour beaucoup sans nom, rende impossible leur dénombrement exhaustif. Il n'est pas indifférent de constater que deux secteurs lui apparaissent particulièrement sujet à la multitude insulaire : la mer Égée, telle qu'elle est dessinée sur les cartes marines - cet espace même qui fera le sujet du Liber insularum archipelagi de Cristoforo Buondelmonti - et l'Orient décrit par les voyageurs ${ }^{20}$. Finalement, le livre d'îles, tel qu'il apparaît avec ces deux auteurs, est l'aboutissement logique d'une longue tradition de catalogues insulaires. L'émergence de l'île comme sujet en soi accompagne les mutations qui affectent l'image du monde au XIV siècle, conséquences des voyages entrepris depuis le XIII ${ }^{\mathrm{e}}$ siècle et d'un regard nouveau porté sur les espaces maritimes grâce à la diffusion des cartes marines.

De la nature et de la fonction des îles

12 Limitée par l'eau, une frontière mouvante, l'île est par essence difficile à localiser. Son instabilité tient à sa nature même. Dans une géographie qui cherche à décrire l'immuable, l'île est soumise au changement: elle naît et disparaît. Le thème de la naissance de l'île - comme celui de son rattachement au continent - est présent dans le livre II des Histoires naturelles de Pline. Au $\mathrm{XII}^{\mathrm{e}}$ siècle, dans la Topographia Hibernica, Giraud de Barri expose une théorie contraire sur la naissance des îles : elles sont nées longtemps après le Déluge, d'une lente montée des eaux qui auraient isolé certaines terres des continents ${ }^{21}$. Ce scénario logique a le mérite d'expliquer la présence des animaux, qui ne saurait se comprendre autrement et dont on examinera plus loin le lien avec la nature insulaire. Il n'abandonne pas pour autant le thème des apparitions soudaines et merveilleuses, comme dans le récit sur l'île Phantastica, qui émerge brusquement au large des Orcades ${ }^{22}$.

Ce caractère mouvant explique l'abondance des prodiges insulaires, décrits surtout à partir du XII ${ }^{e}$ siècle. La Topographia Hibernica en est un témoignage éloquent. Ce goût pour le merveilleux insulaire, qui s'affirme au XII siècle, ne peut se réduire à un effet de l'imaginaire médiéval, particulièrement sensible à l'étrange, à l'inexpliquable ou à l'admiration des œuvres de Dieu, et qui trouverait une place particulière dans les îles atlantiques du fait de l'indigence des connaissances à leur sujet. Giraud de Barri (comme Gervais de Tilbury, l'auteur des Otia imperialia) combine recherche de la nouveauté, qui doit être à la fois plaisir de l'esprit et renouvellement des autorités, et affirmation du merveilleux occidental, qui doit être en mesure de rivaliser avec l'Orient fabuleux $^{23}$. Mais la fascination pour les prodiges est aussi le reflet d'interrogations savantes et de confrontations au concret. Si le merveilleux, dans la Topographia comme 
dans d'autres textes, concerne plus particulièrement les îles océaniques, cela tient aussi à une représentation de l'ordre naturel: dans ces espaces lointains, qu'ils soient atlantiques ou orientaux, le cours de la nature ne s'exprime pas tout à fait comme dans le cœur du monde connu, ainsi que le suggère Giraud à propos de l'Irlande :

L'Irlande est si éloignée du reste du monde - elle forme presque un autre univers qu'elle semble presque, par les phénomènes inconnus du cours ordinaire de la nature, être un réceptacle singulier de trésors naturels, où elle a mis en réserve ses secrets les plus précieux et les plus remarquables ${ }^{24}$.

Et dans le livre II de la Topographia Hibernica, qui contient les prodiges d'une nature joueuse, Giraud tisse le fil d'une observation attentive du phénomène des marées, renvoie vers l'imaginaire l'existence de l'île de Thulé, observe le saut des saumons, tout en dressant un catalogue de prodiges insulaires et de récits merveilleux issus de la tradition orale et écrite. Le merveilleux entretient donc des rapports complexes avec l'imaginaire et le rationnel. Les prodiges demeurent des manifestations de la nature ${ }^{25}$, et par conséquent, sujets de récits divertissants mais aussi objets de connaissance.

Un trait de l'insularité réside dans les particularités de la faune, souvent caractérisée par l'absence d'animaux nuisibles connus sur le continent. Solin mentionne ainsi certaines îles méditerranéennes où ne vit aucun reptile. Isidore de Séville explique le nom de l'île Tanatos par la mort soudaine de tout serpent apporté sur son sol ${ }^{26}$, signale l'absence de reptiles et d'abeilles ainsi que la rareté des oiseaux en Irlande ${ }^{27}$. Il présente la Crète comme abritant de nombreuses chèvres mais aucune bête nuisible. Il existe donc un lien entre la nature insulaire et les animaux qui y vivent. Si Isidore se borne à le constater, Giraud de Barri, qui reprend le topos de l'absence de reptiles en Irlande, tâche de l'expliquer. Il se fait l'écho d'une légende relative à saint Patrick qui, au moment d'entreprendre l'évangélisation de l'île, en aurait chassé les reptiles venimeux. Mais, ajoute-t-il, ce n'est là qu'illusion d'explication puisque de son temps encore, tout serpent apporté sur l'île meurt immédiatement: il faut donc en conclure que saint Patrick n'a rien à voir à l'affaire, et qu'il s'agit là d'une particularité de la nature heureuse et fertile de l'Irlande. Une controverse relative à l'île de Man illustre cette spécificité irlandaise. D'après Giraud, les Anciens s'interrogeaient sur son appartenance à la Bretagne ou à l'Irlande, en raison de sa situation exactement à mi-chemin des deux îles; la dispute fut tranchée par la présence de reptiles venimeux, attestant sans conteste une nature similaire à celle de la Bretagne ${ }^{28}$. L'éloignement et les propriétés de l'Irlande influent aussi sur ses habitants. Giraud justifie sa conquête par la barbarie des Irlandais qui, vivant sur une terre éloignée des peuples policés, ne connaissent que les coutumes barbares dans lesquelles ils ont été élevés et qui constituent comme une « seconde nature $»^{29}$.

16 Cette spécificité naturelle, accompagnée par l'éloignement géographique, concourt à situer dans les îles les entrées de l'au-delà (Purgatoire de saint Patrick en Irlande, île du Purgatoire située dans l'hémisphère inférieur par Dante) ou à en faire le séjour d'une humanité différente. Ce dernier point est excellemment illustré par les jugements de Giraud sur les Irlandais, les discours de Pétrarque sur les habitants des îles Fortunées ou les monstres et autres prodiges qui peuplent les îles du pourtour océanique sur les mappemondes médiévales.

Îles et découvertes

17 Par leur aspect mouvant et insaisissable, les îles sont amenées à jouer un grand rôle dans l'élargissement progressif du monde, notamment à partir du xIV siècle $^{30}$. La 
représentation la plus courante du monde connu est celle d'une masse de terre divisée en trois parties - Asie, Europe, Afrique - encerclée par l'immense océan, siège d'une multitude insulaire dont témoignent des voyageurs comme Marco Polo ou Odoric de Pordenone. Certaines de ces îles matérialisent les limites du monde : l'ultima Thulé des Anciens au Nord-Ouest, Gades (île du détroit de Gibraltar) ou les îles Fortunées pour le Sud-Ouest, Taprobane pour le Sud-Est ${ }^{31}$. Ces îles sont attestées par l'expérience livresque, éprouvée par le témoignage des voyageurs. Il arrive aussi que la lecture des textes mette en évidence les apories de la représentation du monde. Les discussions sur la réalité de Thulé, qui marque la limite du monde occidental pour les Anciens, en constitue un cas remarquable. À deux siècles de distance Giraud de Barri et Pétrarque la renvoient vers l'inconnu océanique. Dans les deux cas, le processus est strictement livresque : c'est la confrontation des données savantes qui conduit les deux auteurs à repousser Thulé au-delà des limites connues du monde, éloignant de la sorte les frontières de l'orbis ${ }^{32}$. La plupart du temps les îles lointaines de la culture géographique sont l'objet des découvertes, comme le montre l'épisode des Canaries. L'invention de ces dernières, connue très rapidement dans le monde des lettrés italiens, a pour première conséquence leur identification avec les îles Fortunées des Anciens ${ }^{33}$. Le pape intervient très vite dans la découverte en concédant les Canaries à un prince francoespagnol, Louis de la Cerda. Dans une lettre où ce dernier lui fait hommage pour son nouveau royaume, il est fait mention de ces îles, auxquelles on attribue des noms tirés de la lecture de Pline et de Martianus Capella. Louis de La Cerda s'y reconnaît vassal du pape «tant pour ces îles que pour d'autres", montrant ainsi que l'on pensait probable la découverte de nouvelles îles. Ce processus des découvertes n'est pas seulement intégration progressive de l'inconnu dans le connu mais surtout extrapolation du possible sur le fondement du livresque. Il est confirmé par les toponymes des cartes marines: dès 1339, Angelino Dulcert représente les Canaries en dessinant deux archipels (Insulle sancti Brendani sive puelarum, Primaria, Capracia, Canaria ; plus au sud, Insula de Lanzaratus Maracelus, Vegimari, La Forteventura). Sur les cartes postérieures, qui intègrent progressivement les Açores et Madère, l'appellation des îles se fait selon les mêmes modalités: toponymes d'origine antique (Canaria, Capraria, Fortunata); toponymes renvoyant à la culture médiévale (insula sancti Brendani siue puelarum; insula deserta, île de l'enfer); toponymes nouveaux comme l'« insula de Lanzarotus Marocelus» ou l'île Brazil. Ce dernier vocable est particulièrement remarquable: sa première attestation se trouve dans le De Canaria de Boccace où il désigne un bois tinctorial inconnu assimilé à du brésiil ${ }^{34}$. Puis, on le voit apparaître sur les cartes pour nommer une île à la localisation si incertaine que les cartographes dessinent parfois deux îles Brasil ${ }^{35}$. Chacun connaît la destinée ultérieure du toponyme. Cette juxtaposition de vocables savants et de noms nouveaux signale un rapport entre livresque et expérience qui n'est pas fondé sur une opposition entre vrai et faux, et qui n'a pas seulement pour résultat de repousser les limites du non-connu. Il s'agit d'un mode de penser l'espace. Le livresque est aussi réel que le résultat de l'exploration: l'expérience conforte le savoir reçu et les connaissances savantes rendent pensable le possible. C'est dire si la conception d'un monde connu entouré d'un océan aux îles innombrables, pour une grande part inconnues et instables, s'est révélée opératoire. C'est dire aussi la place décisive occupée par le xiv siècle dans l'histoire des découvertes, non seulement parce que ce serait là le temps des prémices, mais parce que c'est le moment où un nouveau rapport entre livresque et réalité, entre espace antique et espace moderne, émerge, conduisant à interroger différemment le réel, 
selon des processus plus complexes qu'une simple opposition entre autorité et expérience, imaginaire et réel. Dans cette histoire, les îles sont au premier plan.

Les îles sur les cartes

18 La représentation des îles sur les cartes rencontre des difficultés liées à l'exercice cartographique. Au moment de dessiner les îles, le cartographe doit affronter la question de la localisation, étroitement liée à l'attribution des toponymes. Jusqu'au XIII ${ }^{\mathrm{e}}$ siècle, la grande majorité d'entre eux sont issus de la tradition antique. Dans les textes comme sur les cartes, les îles ont une localisation incertaine. Qu'on en juge par celle des îles Eonee sur la carte de Hereford. Situées par Pline et par Solin dans le flou lointain du grand Nord, elles sont placées sur la carte de Hereford à l'est, sous l'effet d'une probable confusion avec « Eos » ou " Eous », qui signifie « à l'est » ${ }^{36}$. La carte représente des îles qui n'existent pas et qui ont pour fonction d'abriter une légende - au sens cartographique du terme, comme c'est le cas pour le détroit de Gibraltar et les îles Fortunées ${ }^{37}$. Pour autant, l'arbitraire du cartographe est limité par l'existence d'un certain nombre de conventions qui expriment des choix propres à la représentation cartographique. 3778/CARTE2

\section{Carte de Corfou}

(Liber insularum archipelagi de Cristoforo Buondelmonti, $\mathrm{BnF}$ fo $43 \mathrm{r}^{\circ}$ )

Les îles sont présentes dans l'anneau océanique qui entoure le monde - cet anneau, faut-il le rappeler, est une convention cartographique - en Méditerranée et en mer Rouge. Elles peuvent être colorées. Le souci est d'esthétique - une mappemonde relève de l'enluminure - et de lisibilité - il faut voir l'île au sein de la mer. Le déploiement des îles, parfois sans identité, dans l'anneau océanique, est aussi l'expression graphique de l'incommensurable insulaire, comme on le voit sur certaines cartes accompagnant le Commentaire de l'Apocalypse de Beatus. Une autre caractéristique de la représentation consiste à augmenter la taille des îles au point que sur beaucoup de cartes, la Méditerranée est surtout un espace insulaire. Il importe en effet au cartographe de signaler l'existence de ces îles, et par conséquent, d'en grandir le dessin et d'en schématiser le tracé conformément aux données textuelles. De la sorte, elles sont immédiatement identifiables. Ainsi, sur la carte d'Hereford, la Sicile a forme de triangle, tandis que la Crète est suffisamment grande pour y dessiner le labyrinthe. Enfin, sur cette même carte, l'ordonnance du monde insulaire est perceptible par la régularité presque rigide de leur disposition. Il est piquant de constater que les cartes de la fin du Moyen Âge et de la Renaissance, réputées plus justes parce qu'elles donnent une image du monde plus conforme à la nôtre, reprennent ces conventions : même recours possible à la couleur, même disproportion de la taille par rapport au continent, avec une propension à dessiner une indentation du littoral qui donne une fausse impression d'exactitude ${ }^{38}$. Entre les cartes dites de Beatus et l'atlas Miller ( $\mathrm{xvI}^{\mathrm{e}}$ siècle), la distance ne réside pas tant dans le progrès de la représentation que dans les fonctions qui ont présidé à leur réalisation.

Pour autant, on ne saurait ignorer l'évolution dans la représentation des îles, qui se marque en particulier par l'émergence d'une cartographie proprement insulaire, illustrée par le travail de Buondelmonti. Bien avant le $\mathrm{xv}^{\mathrm{e}}$ siècle, des schémas d'îles ont été dessinés, notamment dans les marges des manuscrits géographiques. Dans un exemplaire $\mathrm{du} \mathrm{x}^{\mathrm{e}}$ siècle des Collectanea de Solin, un scholiaste a réalisé des croquis de la 
Sicile, de la Sardaigne et de la Crète, complétés des passages qu'Orose a consacrés à ces mêmes îles dans les Histoires contre les Païens ${ }^{39}$. Ces schémas sont des illustrations graphiques du texte, qui montrent les difficultés à représenter l'île : si la Sicile est de forme triangulaire, les autres îles sont toutes représentées par une ellipse. Une enquête dans les manuscrits permettrait sans aucun doute d'en trouver d'autres témoignages ${ }^{40}$. Dans l'exemplaire de la Topographia Hibernica ( $\mathrm{XII}^{\mathrm{e}}$ siècle) ayant appartenu à Pétrarque, on trouve également un schéma des îles de Bretagne et d'Irlande, illustration et résumé graphique du texte, comme en témoigne le repentir du dessinateur qui a gommé une partie du trait pour représenter l'Irlande plus grande et plus resserrée en son centre. Les données des chapitres 1 à 3 y sont fidèlement rappelées : orientation identique et position parallèle des deux îles, Bretagne deux fois plus grande que l'Irlande, qui est elle-même resserrée en son milieu.

Cependant, c'est surtout à partir du XIV siècle que l'on trouve les premiers témoins d'une représentation autonome des îles, en relation avec l'émergence d'une cartographie régionale. Il s'agit là d'un domaine encore assez peu fréquenté par les historiens de la cartographie. Quelques exemples, dans le domaine anglais, laissent penser que de telles représentations étaient plus nombreuses qu'on ne le croit communément et - peut-être aussi plus précoces ${ }^{41}$. En ce sens, le travail cartographique réalisé par Cristorofo Buondelmontiti ${ }^{42}$ paraît moins novateur, d'autant qu'il s'inscrit dans une tradition bien avérée qui fait de la carte et du texte, placés côte à côte dans les manuscrits, deux modes de représentation indispensables pour qui entend comprendre le monde. Ainsi, à la même époque, le commentaire de Guglielmo Capello au Dittamondo de Fazio degli Uberti ou la Sfera de Leonardo Dati témoignent de la diffusion d'œuvres fondées sur l'usage conjoint et raisonné du texte et de la carte.

Les cartes du Liber insularum archipelagi, résultat probable de l'influence de la cartographie marine et de l'essor de la cartographie régionale, illustrent les grandes tendances de la cartographie des $\mathrm{XIV}^{\mathrm{e}}-\mathrm{XV} \mathrm{v}^{\mathrm{e}}$ siècles. Elles se caractérisent par l'absence d'échelle, le découpage exagéré du littoral, et le souci de souscrire à un ensemble de conventions (voir carte). Les îles sont en général dessinées dans un cadre qui les isole dans la page manuscrite, parfois dans un contexte régional constitué par le dessin de la côte. Le relief est représenté. Montagnes et plaines sont coloriées de deux couleurs différentes et structurées par le réseau hydrographique soigneusement dessiné. Crisotoforo Buondelmonti a lui-même donné des indications relatives aux couleurs qui ont pour fonction de faciliter la lecture : les eaux doivent être vertes, les montagnes marron, les plaines blanches ${ }^{43}$. Enfin, la carte porte les lieux illustres, villes, églises, monastères, ruines. Il s'agit de dessiner les éléments constitutifs de la perception du paysage développée par l'humanisme: un paysage perçu et analysé en fonction des marques de l'occupation humaine, passée et présente.

$\mathrm{Si}$, dès les premiers siècles du Moyen Âge, les îles tiennent une place particulière dans l'économie des traités de géographie, cela est dû autant à l'epistémé de la géographie médiévale, qui classe volontiers les êtres géographiques par catégorie, qu'au souci de leur donner place dans des descriptions fondées sur un découpage ordonné du monde. Sans doute, la nature particulière de l'espace insulaire n'est pas ignorée - un parcours dans la littérature géographique carolingienne permettrait d'affiner notre propos. Cependant, la reconnaissance de la spécificité insulaire s'affirme au XII ${ }^{\mathrm{e}}$ siècle, en relation avec le goût du prodige, mais aussi comme résultat d'un regard nouveau porté sur le réel, qui fait une place plus grande au concret. Surtout, la connaissance du milieu 
insulaire est fondée sur une culture essentiellement livresque : il en est encore ainsi au moment où l'Occident s'ouvre aux espaces atlantiques. Mais on aurait tort de caractériser l'inventaire du monde en termes d'opposition entre ce qui est découvert par le fruit de l'aventure de l'explorateur, et le travail du géographe et du cartographe de cabinet, encore soumis à la dictature des connaissances textuelles pour une bonne part fabuleuses. Si les cartographes du $\mathrm{XV}^{\mathrm{e}}$ siècle dessinent autant d'îles imaginaires sur les cartes, ce n'est pas seulement pour combler un blanc, ou pour faire joli : les porter sur la carte confirme leur existence et la possibilité d'y accoster un jour. Ce caractère particulier que revêt la vérité textuelle au Moyen Âge se découvre aussi dans la représentation cartographique des îles: celle-ci répond à des conventions et à des usages précis dont la fonction principale est de permettre une lecture sensée de la carte. Par conséquent, il ne faut pas interpréter l'évolution du dessin des îles dans le sens du progrès d'une cartographie qui se voudrait plus fidèle au réel mais comme une adaptation progressive à des usages renouvelés, comme le montrent les dessins d'îles qui accompagnent le Liber insularum archipelagi de Cristoforo Buondelmonti.

\section{NOTES}

1. Voir en dernier lieu D. Lecoq, « îles du dedans, îles du dehors. Les îles médiévales entre le réel et l'imaginaire ( $\mathrm{VII}^{\mathrm{e}}-\mathrm{XIII}{ }^{\mathrm{e}}$ siècles) », dans Les îles, du mythe à la réalité, éd. M. Pelletier, Paris, 2002, p. 18-51; pour un parcours sur les îles dans la littérature antique et médiévale, voir J. M. Montesdeoca Medina, «Del enciclopedismo grecolatino a los islarios humanistas. Breve historia de un género ", Revista de Filología, 19, 2001, en particulier p. 242-253 pour le Moyen Âge.

2. F. Lestringant, Le livre des îles. Atlas et récits insulaires de la Genèse à Jules Verne, Genève, 2002, p. 24.

3. À la fin du livre II, Pline l'ancien aborde la question insulaire mais dans un exposé général sur les phénomènes naturels extraordinaires auquel est rattaché un développement sur la naissance et la disparition des îles. Il ne s'agit pas d'un mode spécifique de traitement des îles.

4. Par exemple la Sardaigne, Pline, III, 84 ou la Corse, III, 80.

5. Pomponius Mela, De Chorographia, A. Silberman éd., respectivement II, 97 et III, 46.

6. Orose, Histoires contre les Païens, M.-P. Arnaud-Lindet éd. et trad., Paris, 1990, I, $2,95$.

7. «Les îles sont dénommées ainsi parce qu'elles sont dans le sel, c'est-à-dire dans la mer », Isidore de Séville, Etymologiae sive originum libri xx, W. M. Lindsay éd., Oxford, 1911, XIV, 6, 1.

8. "Commune est insulis ut promineant. Inde et loca earum promuntoria dicuntur ; Montes sunt tumores terrarum altissimi, dicti quod sint eminentes ", ibid., XIV, 7, 1 et XIV, 8, 1. Une telle remarque a déjà été faite par P. Gautier Dalché, « La montagne dans la description "géographique" au Moyen Âge », Cl. Thomasset, D. James-Raoul dir., $L a$ Montagne dans le texte médiéval. Entre mythe et réalité, Paris, 2000, p. 103-104.

9. Un autre texte écrit dans l'Antiquité tardive, la Cosmographia de Julius Honorus (après 376), remanié plus tard par un auteur désigné sous le nom de Pseudo-Aethicus, 
se présente sous la forme d'une liste de toponymes - fleuves, montagnes, mers, peuples, villes, îles. A. Riese éd., Geographi latini minores, Heilbronn, 1878, p. 21-55.

10. Hugues de Saint-Victor, $L a$ « descriptio mappe mundi » de Hugues de Saint-Victor, texte inédit avec introduction et commentaire, P. Gautier Dalché éd., Paris, 1988.

11. Ibid., p. 106.

12. "In orientali parte occeani, inter subsolanum et eurum, sita est insula Taprobana... Inter eurum et euroaustrum est insula Malius nomine... inter euroaustrum et austrum est insula Adamantus... », ibid., p. 134-135.

13. "Prima igitur insula est Gades, sita in ipso ostio unde mare Magnum ab occeano diriuatur, inter Auennam Hispanie montem a septentrione et Calpem montem Africe ab occidente... Post quas Ebusus insula et ciuitas eiusdem nominis. Quam sequitur Balearis maior et alia minor... ", ibid., p. 136, 1. 106.

14. "Inter has insulas que numero XLVIIII sunt maiores quinque existunt, plurimas habentes ciuitates siue etiam prouincias ", ibid., p. 137, 1. 129-130.

15. Ibid., p. 136, l. 123. Comme le fait remarquer l'éditeur, une explication logique peut être donnée à cette erreur : pour Brundusium et Apulia, la mappemonde inscrivait ces noms dans la mer ; pour Venetia, placée plus loin parmi les îles de l'Adriatique, il s'agit plus probablement d'une reconnaissance de sa nature lagunaire. Voir ibid., p. 162-163. La carte d'Hereford représente elle aussi Venetia comme une île.

16. Barthélemy l'Anglais, De proprietatibus rerum, Francfort, 1609, p. 650.

17. Cf. P. Gautier Dalché, Carte marine et portulan au XII siècle. Le Liber de existencia rivierarum et forma maris nostri mediterranei, Rome, 1995 (Collection de l'École française de Rome, 203).

18. Sur l'usage d'une carte marine par Riccobaldus de Ferrare, voir P. Gautier Dalché, «Riccobaldus de Ferrare géographe. À propos de l'édition du De locis orbis et insularum et marium ", Sacris Erudiri, 30, 1987-1988, p. 409-434 ; sur la diffusion des portulans et cartes marines en Italie au XIV ${ }^{\mathrm{e}}$ siècle, voir N. Bouloux, Culture et savoirs géographiques en Italie au XIV siècle, Turnhout, 2002 (Terrarum orbis, 2), p. 88-105.

19. Marino Sanudo, Liber secretorum fidelium crucis super Terrae sanctae recuperatione et conservatione, Gesta dei per Francos, II, J. Bongars éd., Hanovre, 1611, p. 287.

20. «Tot insuper tanteque in quolibet oceano emisp[eri nostri] sunt insulae quarum etiam notitiam non habemus deve quibus sine nominibus scribunt autores, quod nedum omnes, sed minimam earum partem ad plenum puto impossibile sit amplecti. Quot solum in Agios pelago, parva maris Egei parte, designant navigationis periti innumerabiles, enim earum figuras inter Acaiam Cretamque interque Rodum ac Sergest[um] esse demonstrant [...] Marcus Polus venetus scribit tantam insularum multitudinem in India esse quod foret eas earumque conditiones difficillime recitari ; Quinque milia insularum sub regimine Tartarorum regis fore dicitur, Odorigo referenti si credimus », Domenico Silvestri, De insulis et earum proprietaibus, C. Pecoraro éd., Atti della accademia di scienze lettere e arti di Palermo, série 4, vol. 14, part. 2, 1953-1954, fasc. 2, p. 30-31.

21. Giraud de Barri, Topographia Hibernica, II, XVI, J.-F. Dimock éd., Londres, 1867 (réimpr. 1964), Giraldi Cambrensis opera, vol. 5 (Rerum britannicarum medii aevi scriptores). 22. Ibid., II, 12, p. 94-95.

23. Ces deux aspects sont développés dans les préfaces de la Topographia Hibernica. 24. "Quae videlicet Hibernia, quanto a cetero et communi orbe terrarum semota, et quasi alter orbis esse dignoscitur, tanto rebus quibusdam solito naturae cursui incognitis, quasi peculiaris ejusdem naturae thesaurus, ubi insignia et pretiosiora sui 
secreta reposuerit, esse videtur » (Top. Hib.), I, 2, p. 23. Voir aussi : « Quaelibet nimirum regiones, insulae praesertim et partes a centro remotissimae, propriis quibusdam prodigiis pollent » (ibid., II, préface, p. 75).

25. Je rappelle ici la définition des mirabilia par Gervais de Tilbury : « Mirabilia uero dicimus que nostre cognicioni non subiacent, etiam cum sunt naturalia ; sed et mirabilia constituit ignorantia reddende rationis quare sic sit », Otia imperialia. Recreation for an emperor, S. E. BANKS et J. W. BINNS éd., Oxford, 2002, p. 558 (Oxford Medieval Texts).

26. Étym., XIV, VI, 3.

27. Ibid., XIV, VI, 6.

28. Top. Hib., II, 14, p. 97.

29. Top. Hib., III, 10, p. 153.

30. Voir F. Lestringant, op. cit., p. 13-15.

31. Par exemple, Gervais de Tilbury, «Verumptamen Orcades insulas, Islandiam, Gothlandiam, et Vltimam Tylen intra fines Europes censemus », Otia imperialia, éd. cit., p. II, 11, p. 326. Voir aussi Pétrarque, Fam. XI, 8, 35 et IX, 13, 8.

32. Cf. N. Bouloux, op. cit., p. 266-273.

33. Voir la traduction et le commentaire de ce texte dans le présent numéro.

34. Ibid., p. 9-10.

35. Voir par exemple l'atlas de Grazioso Benincasa réalisé en 1467, BnF, Cartes et Plans, Rés. GeDD 1988, fo 2.

36. La carte de Hereford est désormais accessible grâce à la reproduction, l'édition des légendes et leur commentaire par S. D. Westrem, The Hereford Map, Turnhout, 2003

(Terrarum orbis 3). Pour les îles Eonee, voir p. 20-21, nos 35 et 39.

37. Voir carte de Hereford, p. 388, nº 987 ; p. 402, nº 1025 ; p. 426, nº 1091.

38. W. E. Washburn, « The form of islands in fifteenth, sixteenth and seventeenthcentury cartography ", dans Géographie du Monde au Moyen Âge et à la Renaissance, M. Pelletier éd., Paris, 1989, p. 201-206.

39. Vatican, BAV, Vat. lat. 3342 , fo $27 v^{\circ}$ : Sardaigne; fo $29 \mathrm{r}^{\circ}$ : Sicile ; fo $73 \mathrm{v}^{\circ}$ : Crète. Au $f^{\circ} 103 r^{\circ}$, un passage d'Orose consacré à la Bretagne est ajouté en marge, en face de la description par Solin ; au fo $203 v^{\circ}$, une ellipse ratée ou inachevée devait représenter Taprobane.

40. Voir par exemple le croquis de la Sicile dans un manuscrit de Solin datant du xiv $^{\mathrm{e}}$ siècle (BAV, Ross. 228, fo 22).

41. Voir P. D. A. HARVEY et R. A. SKELTON, Local maps and plans from medieval England, Oxford, 1986 : île de Thanet.

42. Le Liber insularum archipelagi présente une tradition manuscrite complexe, qui rend aujourd'hui encore difficile son étude. Sur tous les problèmes liés à cette œuvre et l'abondante bibliographie sur l'auteur et son œuvre, voir en dernier lieu, G. Ragone, « Il Liber insularum archipelagi di Cristoforo dei Buondelmonti : filologia del testo, filologia dell'imagine ", dans Humanisme et culture géographique à l'époque du concile de Constance. Autour de Guillaume Fillastre (acte du colloque de l'Université de Reims, 18-19 novembre 1999), D. Marcotte éd., Turnhout, 2002 (Terrarum orbis 3).

43. «Ea propter ut cuncta comprehendas, in nigro montes, in albo planities, in viridi aquae panduntur manifeste », Cristoforo Buondelmonti, Liber insularum archipelagi, éd. L. De Sinner, Leipzig-Berlin, 1824, p. 53-54. 


\section{RÉSUMÉS}

Espace essentiellement discontinu, les îles trouvent difficilement place dans les descriptions du monde, fondées depuis Orose sur la localisation des régions par leur contiguïté. Elles sont donc le plus souvent rassemblées sous la forme de catalogue à la fin des descriptions. Au XII ${ }^{\mathrm{e}}$ siècle, leur caractère mouvant explique leur rôle dans l'essor du merveilleux et dans les réflexions conduites par les clercs sur le monde naturel. Nombreuses dans l'océan qui entoure le monde connu, elles accompagnent à partir du XIv ${ }^{\mathrm{e}}$ siècle l'inventaire du monde. Quant à leur représentation cartographique, elle suit les grandes lignes de l'évolution de la cartographie médiévale.

Islands in the Geographical Descriptions and Maps in the Middle Ages. Since Orose the depictions of the world have been founded on the localization of the regions through their contiguity and therefore, being an essentially discontinuous space, it is hard to give islands a place in them. Actually, they are more often than not collected in a catalogue at the end of those depictions. In the 12th century, their part in the blossoming of the fantastic element and the reflection of the scholars about the natural world is explained by their changing characteristics. Being numerous in the ocean that surrounds the known world, they have been part of the world inventory since the 14th century. As for their geographic representation, it has followed the general evolution of the medieval cartography.

\section{INDEX}

Keywords : islands, Marvelous, discovery, space, cartography

Mots-clés : espace, représentation cartographique, Merveilleux, découvertes, îles

\section{AUTEUR}

\section{NATHALIE BOULOUX}

Université François Rabelais, 3, rue des Tanneurs, UFR Arts et Sciences humaines, Département d'Histoire, BP 41003, F-37041 Tours Cedex 01 